\title{
Linear Encoder-Decoder-Controller Design over Channels with Packet Loss and Quantization Noise
}

\author{
S. Dey, A. Chiuso and L. Schenato
}

\begin{abstract}
In this paper we consider the problem of designing coding and decoding schemes for linear control design of a scalar unstable stochastic linear system in the presence of a wireless communication channel between the sensor and the estimator. In particular, we consider a communication channel which is prone to packet loss and includes quantization noise due to its limited capacity. We first study the case of perfect channel feedback, where the transmitter is aware of the quantization noise and the packet loss history of the channel. We show that in this case, the optimal strategy among all possible linear encoders corresponds to the transmission of the Kalman filter innovation (the difference between the filtered state estimate at the transmitter and the predicted state estimate at the receiver) similarly to the differential pulse-code modulation (DPCM). Although the critical Signalto-Quantization Noise Ratio (SQNR) required for stabilizing the system is the same for innovation forwarding as well as measurement forwarding at the transmitter, the latter is strictly suboptimal in terms of control performance. For the case of imperfect feedback, we assume that the channel feedback or acknowledgement is randomly lost with a certain erasure probability, rendering the transmitter ignorant of the control action taken by the receiver and subsequently applied to the plant. We propose several heuristic strategies for a suboptimal Kalman filter design at the transmitter based on estimation of the channel feedback status and compare their performances via numerical simulation studies.
\end{abstract}

\section{INTRODUCTION}

The interplay between control stability and communication channels non-idealities has attracted considerable attention in past decade, mainly driven by the success of wireless communication and its penetration into automation and control applications. From a theoretical perspective, we have witnessed the convergence of control theory, communication theory and information theory which have obtained remarkable and interesting results in terms of the ultimate performance limitations which take into account both the dynamical systems characteristic, typically their unstable eigenvalues and non-minimum phase zeros, and the channel characteristic, typically its capacity [1], [2], [3], [4], [5], [6], [7], [8], [9], [10].

Nonetheless, there are still important open questions that need to be answered. For example most of the results are obtained for scenarios in which the transmitter has

This work is supported by by the European Community's Seventh Framework Programme [FP7/2007-2013] under grant agreement n. 257462 HYCON2 Network of excellence and by the Swedish Research Council grant Dnr 621-2013-5395.

S. Dey is with the Dept. of Signals \& Systems, Uppsala University, Uppsala, SE 75121 Sweden, email: Subhra.Deyesignal.uu.se, while A. Chiuso and L. Schenato are in the Department of Information Engineering, University of Padova, Via Gradenigo 6/b, 35131 Padova, Italy, email: $\{$ chiusolschenato\}@dei.unipd.it full knowledge of what happened over the channel in the past (perfect channel feedback), which guarantees several separation principles both in terms of controller/observer design and of source/channel coding, see e.g. [11] for early references. Differently if the channel feedback is not present or it is "imperfect", very few results are available and mainly based on heuristics [12], [13], [14], [15], [16]. Another difficulty raises when both packet loss and quantization are considered simultaneously. In fact, if these two limitations are considered separately, the resulting optimal strategies can be quite different. For example, in a scenario with packet loss only, Gupta et al. [17] showed that the optimal strategy is to sent the estimate of the state over the channel, which does not even require channel feedback to the transmitter and it is therefore quite attractive. Differently, if only rate limitation, i.e. the maximum number of symbols that can be transmitted per unit of time, is considered, then differential coding results to be the optimal strategy as in differential pulse-code modulation [18]. Obviously, this strategy is rather different from the one that transmits the complete state estimate. Recently, in [19] we have shown that a strategy inspired by DPCM, is optimal in the context of remote estimation when considering both packet loss and quantization limitations for a scalar stable system when full channel feedback is present. However, the optimal strategy in the presence of imperfect channel feedback remains elusive and only sensible heuristic have been proposed in [19]. In the context of closed loop unstable control system, simultaneous analysis of packet loss and quantization has been studied in [20] assuming that the transmitter simply forwards a quantized version of the raw measurement.

In this work we extend the results of [19] and [20] by considering the possibility to pre-process the raw measurement at the transmitter. We show that the optimal strategy when full channel feedback is available at the transmitter is to send the difference from the estimated state at the transmitter and the predicted state at the receiver as in [19] and to build a Kalman filter and a state feedback with constant gain at the receiver as in [20]. However, although the performance is improved as compared to strategy proposed in [20], the stability region is the same. In the imperfect channel scenario, we propose a number of heuristics similarly to those proposed in [19]. However, differently from remote estimation, in closed loop systems the effect of packet loss between the transmitter and the controller appears implicitly in the measurements observed at the transmitter even in the absence of any channel feedback. As so, we propose an on-line strategy to estimate whether a packet has been 
lost or received in the same spirit of [16] and we use it to send a differential signal, which is observed to provide better performance over the other strategies in extensive simulations.

\section{System Model And Problem Formulation}

In this section, we provide the system model of the networked control system under consideration along with the LQG problem formulation.

\section{A. Plant Model}

We consider a discrete-time linear scalar time-invariant unstable plant as given below:

$$
\begin{aligned}
x_{t+1} & =a x_{t}+b u_{t}+w_{t} \\
y_{t} & =c x_{t}+v_{t}
\end{aligned}
$$

where $x_{t}$ is the scalar state, $u_{t}$ is the scalar control input, and $y_{t}$ is the scalar measurement or plant output. $w_{t}, v_{t}$ are also scalar process and measurement noise processes respectively, independent and identically Gaussian distributed with zero mean and variances $\sigma_{w}^{2}$, and $\sigma_{v}^{2}$, respectively. We make the usual assumption that $w_{t}, v_{t}, x_{0}$ are mutually independent. Finally, $a, b$ and $c$ are the state, input and output coefficients, respectively. In our networked control system model, a sensor senses the plant output $y_{t}$ and transmits a suitable signal $s_{t}$ after some pre-processing to a remote estimator/controller over a communication channel. We restrict $s_{t}$ to be a causal linear function of the measurements $\mathcal{Y}_{t} \triangleq\left\{y_{0}, y_{1}, \ldots, y_{t}\right\}$. The remote estimator/controller block constructs a state estimate and design a linear feedback controller which generates the control input $u_{t}$ to be fed back to the plant, see Figure 1. The objective of the control design problem is to find optimal $\left\{s_{t}\right\}$ and $\left\{u_{t}\right\}$ such that the average cost $J=$ $\lim _{T \rightarrow \infty} \frac{1}{T} \sum_{t=0}^{T} \mathbb{E}\left[y_{t}^{2}\right]$ is (finite and) minimized.

\section{B. Communication channel}

We assume that the channel between the sensor (which senses the plant output) and the remote estimator/controller is subject to both bandwidth constraints and packet loss. Thus, the signal transmitted from the sensor is quantized by a quantizer of fixed but sufficiently high rate to produce a quantized signal $s_{t}^{q}$. Under a fine quantization assumption, it has been established by multiple authors including our previous work [19] that the error due to quantization can be represented as an additive white Gaussian noise (AWGN) which is also independent of the input signal, with a variance that is proportional to the variance of the input signal $s_{t}$. Thus, the quantized signal can be written as $s_{t}^{q}=s_{t}+n_{t}$, where $n_{t}$ is an additive white Gaussian noise (AWGN) with zero mean and variance $\frac{\mathbb{E}\left[s_{t}^{2}\right]}{\Lambda}$ (note also that $\mathbb{E}\left[s_{t}\right]=0$ ), where $\Lambda$ is known as the Signal-to-Quantization Noise Ratio (SQNR).

Remark 1: The validity of the additive quantization noise model for high rate uniform scalar quantization has been rigorously shown in [21] for continuous input densities, and see also [22] for similar studies. It has been however shown in these papers as well as many other recent literature such as in [23] that although in principle only high rate quantization theory justifies such an additive white quantization noise model, in practice this model holds as a very good approximation for moderate rate quantization. If fact, it was shown in [19] via numerical simulations that, a uniform scalar quantizer with only 3-4 bits of quantization per sample used to quantize the signal $s_{t}$ provides results that are sufficiently close to the theoretical values based on the additive noise model proposed in this work. Note that in a wireless local area network (WLAN) with orders of megabits per second data rates (even when shared amongst multiple links), it is not unreasonable to expect 3-4 bits per sample with a sampling rate of say $0.1 \mathrm{MHz}$ which is likely to be sufficient for most physical dynamical systems. Thus, this additive white quantization noise model is also suitable for use in practical implementation of estimation over lossy wireless links.

The communication channel is also subject to a packet loss process $\gamma_{t} \in\{0,1\}$, which is modelled as a independent and identically distributed (i.i.d.) Bernoulli process with $P\left(\gamma_{t}=0\right)=\epsilon_{\gamma}$, which is known as the packet loss probability. When $\gamma_{t}=1$, the receiver receives $s_{t}^{q}$ perfectly. But when $\gamma_{t}=0$, the information is lost and the receiver does not receive anything. We assume that the receiver sends a packet acknowledgement signal ACK/NACK back to the transmitter to indicate whether it has received the packet. In the case of full channel feedback, the transmitter has exact knowledge of the packet loss sequence $\left\{\gamma_{t}\right\}$, whereas in the case of imperfect channel feedback, the ACK/NACK packet can also be lost randomly according to another i.i.d. Bernoulli erasure process $\nu_{t}$, which is independent of $\gamma_{t}$. When $\nu_{t}=1$, the transmitter knows the exact value of $\gamma_{t}$, whereas when $\nu_{t}=0$, the transmitter does not know $\gamma_{t}$. We also denote $P\left(\nu_{t}=0\right)=\epsilon_{\nu}$. It is assumed that when the ACK/NACK packet is received, it is decoded correctly, as a 1 bit information can be easily coded with strong error correcting codes for reliable decoding.

The feedback control channel between the receiver (also called the remote estimator/controller) is assumed to be perfect with no delay, packet loss or quantization related losses.

\section{Remote estimator/controller}

The remote estimator receives the intermittent sequence $\mathcal{Z}_{t} \triangleq\left\{z_{0}, z_{1}, \ldots, z_{t}\right\}$, where $z_{t}=\gamma_{t}\left(s_{t}+n_{t}\right)$ and has to produce a control input $u_{t}$. We shall restrict ourselves to linear strategies. In the forthcoming section we shall see that under perfect channel feedback (i.e. when the transmitter knows the loss sequence $\Gamma_{t} \triangleq\left\{\gamma_{0}, \gamma_{1}, \ldots, \gamma_{t}\right\}$ ), the optimal strategy will be the cascade of a constant gain state estimator followed by a constant gain (estimated) state feedback controller. This motivates us to consider, also when no channel feedback is present, a similar constant gain "estimator-controller" structure.

\section{OPTIMALITY OF INNOVATION FORWARDING WITH FULL CHANNEL FEEDBACK}

We now consider a general linear coding-decodingcontroller scheme as follows: 


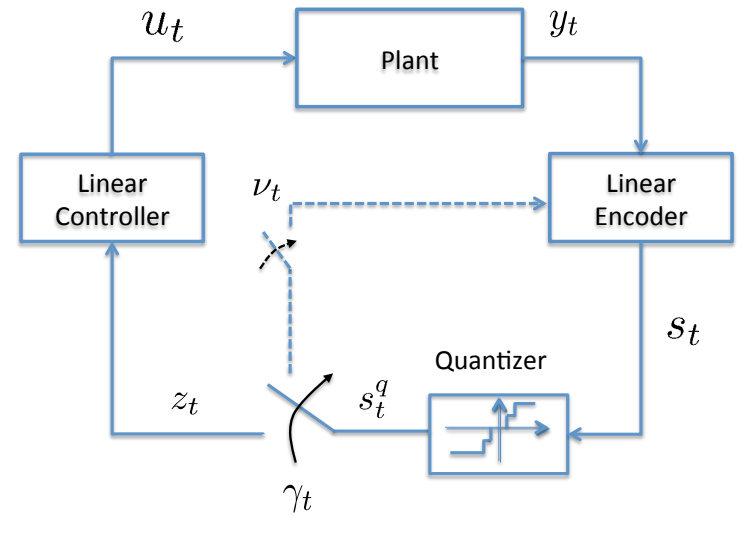

Fig. 1. General scheme of the control system under communication constraints. The sequence $\gamma_{t}$ is fed back to the coder only when channel feedback is available.

1) a linear coding mechanism produces the signal

$$
s_{t}:=\mathcal{L}_{\gamma}\left(\mathcal{Y}_{t}, \mathcal{U}_{t-1}, \mathcal{Z}_{t-1}, \Gamma_{t-1}\right)
$$

where $\mathcal{L}_{\gamma}\left(\mathcal{Y}_{t}, \mathcal{U}_{t-1}, \mathcal{Z}_{t-1}, \Gamma_{t-1}\right)$ is, conditionally on the packet loss sequence $\gamma_{t-1}, \ldots, \gamma_{0}$, a linear operator of its arguments $y_{t}, y_{t-1}, . ., y_{0}$ (the samples to be encoded), $u_{t-1}, . ., u_{0}$ (the past control signal, which can be reconstructed at the transmitter side if channel feedback is available) and $z_{t-1}, . ., z_{0}$ (the past received signals).

2) The signal $s_{t}$ is sent through a lossy and noisy channel (see Section II-B) and produces the received signal $z_{t}$ which can be modeled as

$$
z_{t}=\gamma_{t}\left(s_{t}+n_{t}\right)
$$

where $n_{t}$ is a white noise signal with variance proportional to the variance of $s_{t}$, i.e. $\operatorname{Var}\left\{n_{t}\right\}=\frac{\operatorname{Var}\left\{s_{t}\right\}}{\Lambda}$ and $\gamma_{t}$ is a binary random variable which models the loss events.

3) The controller uses the received signals $z_{t}$ to build the control action at time $t$ as a (conditionally on the packet loss sequence $\left.\gamma_{t}, \ldots, \gamma_{0}\right)$ linear function $\mathcal{C}_{\gamma}$ of the past received signals $z_{\tau}, \tau \in[0, t]$ as well as past control signals $u_{\tau}, \tau \in[0, t)$

$$
u_{t}=\mathcal{C}_{\gamma}\left(\mathcal{Z}_{t}, \mathcal{U}_{t-1}, \Gamma_{t}\right)
$$

Note that, in principle, the conditional linear mappings $\mathcal{L}_{\gamma}$ and $\mathcal{C}_{\gamma}$ are time-varying. The result of this section is summarized in the next theorem. The proof is omitted for space restrictions and can be found in [24]. For convenience of notation we define

$$
\mathcal{I}_{t}^{T x}:=\left\{\mathcal{Y}_{t}, \mathcal{Z}_{t}, \mathcal{U}_{t-1}, \Gamma_{t-1}\right\} \quad \mathcal{I}_{t}^{R x}:=\left\{\mathcal{Z}_{t}, \mathcal{U}_{t-1}, \Gamma_{t}\right\}
$$

which are, respectively, the information set at the transmitter $\left(\mathcal{I}_{t}^{T x}\right)$ and receiver $\left(\mathcal{I}_{t}^{R x}\right)$. Denote the state estimates at the transmitter and receiver as $\hat{x}_{t \mid t}^{t x}=\mathbb{E}\left[x_{t} \mid \mathcal{I}_{t}^{T x}\right]$ and $\bar{x}_{t \mid t}=$ $\mathbb{E}\left[x_{t} \mid \mathcal{I}_{t}^{R x}\right]$.
Theorem 1: Consider the linear model (1) controlled through a lossy and SNR limited channel (3) using a linear encoding as in (2) and linear controller as in (4). The optimal linear quadratic strategy

$$
\left(\mathcal{C}_{\gamma}^{*}, \mathcal{L}_{\gamma}^{*}\right):=\underset{\mathcal{C}_{\gamma}, \mathcal{L}_{\gamma}}{\arg \min } \lim _{T \rightarrow \infty} \frac{1}{T} \sum_{t=0}^{T} \mathbb{E}\left[y_{t}^{2}\right]
$$

satisfies the following:

$$
s_{t}=\mathcal{L}_{\gamma}^{*}\left(\mathcal{I}_{t}^{T x}\right)=\hat{x}_{t \mid t}^{t x}-\bar{x}_{t \mid t-1}
$$

and

$$
u_{t}=\mathcal{C}_{\gamma}^{*}\left(\mathcal{I}_{t}^{R x}\right)=-\frac{a}{b} \bar{x}_{t \mid t}
$$

The state estimator at the receiver is given by

$$
\begin{aligned}
\bar{x}_{t+1 \mid k} & =a \bar{x}_{t \mid t}+b u_{t}=0 \\
\bar{x}_{t+1 \mid t+1} & =\bar{x}_{t+1 \mid t}+k z_{t+1}=k z_{t+1}
\end{aligned}
$$

with $k=\frac{1}{1+\frac{1}{\Lambda}}$.

\section{OPTIMAL LQG COST WiTH FULL CHANNEL FEEDBACK: INNOVATION FORWARDING VS. MEASUREMENT FORWARDING}

As we have seen in the previous section the optimal linear encoding/decoding/control strategy in the full channel feedback case is obtained by sending the state innovation $s_{t}:=\mathbb{E}\left[x_{t} \mid \mathcal{I}_{t}^{T x}\right]-\mathbb{E}\left[x_{t} \mid \mathcal{I}_{t-1}^{R x}\right]=\hat{x}_{t \mid t}^{t x}-\bar{x}_{t \mid t-1}$ and performing a constant gain state feedback control $u_{t}=-\frac{a}{b} \bar{x}_{t \mid t}$. The estimation error $\tilde{x}_{t \mid t}^{t x}=x_{t}-\hat{x}_{t \mid t}^{t x}$ is Gaussian distributed with zero-mean and a steady-state variance $p_{\infty}^{t x}$. It can be shown from standard Kalman filter steady state analysis that $p_{\infty}^{t x}=\frac{\sigma_{v}^{2} \bar{p}_{\infty}^{t x}}{\bar{p}_{\infty}^{x+}+\sigma_{v}^{2}}$ where $\bar{p}_{\infty}^{t x}$ is the steady state prediction error covariance at the transmitter and can be computed as a solution to an algebraic Riccati equation as

$$
\begin{aligned}
\bar{p}_{\infty}^{t x}=\frac{1}{2} & \left(\sigma_{v}^{2}\left(a^{2}-1\right)+\sigma_{w}^{2}\right. \\
& \left.+\sqrt{\left(\sigma_{v}^{2}\left(a^{2}-1\right)+\sigma_{w}^{2}\right)^{2}+4 \sigma_{v}^{2} \sigma_{w}^{2}}\right)
\end{aligned}
$$

For ease of analysis, we henceforth assume that the transmitter Kalman filter has reached its steady state. When the transmitter has full channel feedback, it can also reconstruct a copy of the receiver constant gain Kalman filter (after receiving $\gamma_{t}$ via the ACK/NACK packet), which is given by (8). When the optimal control $u_{t}=-\frac{a}{b} \bar{x}_{t \mid t}$ is used the state update takes the form:

$$
\begin{aligned}
\bar{x}_{t \mid t} & =k \gamma_{t}\left(\hat{x}_{t \mid t}^{t x}-\bar{x}_{t \mid t-1}+n_{t}\right) \\
\bar{x}_{t+1 \mid t} & =a \bar{x}_{t \mid t}+b u_{t}=0
\end{aligned}
$$

where $\bar{x}_{t+1 \mid t}, \bar{x}_{t \mid t}$ are the receiver predicted and filtered state estimates, respectively, and $k$ is the constant gain of the optimal linear filter employed at the receiver. Note that the transmitter sends the innovation signal $s_{t}=\hat{x}_{t \mid t}^{t x}-\bar{x}_{t \mid t-1}=$ $\tilde{x}_{t}-\tilde{x}_{t \mid t}^{t x}$ after quantization, where $\tilde{x}_{t}=x_{t}-\bar{x}_{t \mid t-1}$ is the receiver prediction error. As explained earlier, the effect of quantization is modelled (under high rate quantization) as an additive zero-mean white Gaussian noise $n_{t}$ with variance 
$\sigma_{n}^{2}=\mathbb{E}\left[s_{t}^{2}\right] / \Lambda$. It can be shown following the analysis in [19] that $\mathbb{E}\left[s_{t}^{2}\right]=\mathbb{E}\left[\tilde{x}_{t}^{2}\right]-p_{\infty}^{t x}$. For the subsequent analysis, without loss of generality, we assume $b=1, c=1$ to simplify the expressions. The receiver prediction error follows the dynamics

$$
\tilde{x}_{t+1}=a\left(1-k \gamma_{t}\right) \tilde{x}_{t}+k a \gamma_{t} \tilde{x}_{t \mid t}^{t x}+\left(w_{t}-k a \gamma_{t} n_{t}\right)
$$

Denoting $\mathbb{E}\left[\tilde{x}_{t}^{2}\right]=p$ one can easily see that $\sigma_{n}^{2}=\frac{\left(p-p_{\infty}^{t x}\right)}{\Lambda}$. Note that here the expectation is taken over the noise processes $w_{t}, n_{t}$ and the packet loss sequence $\Gamma_{t}$. Since $y_{t}=x_{t}+v_{t}$ and $\tilde{x}_{t}:=x_{t}-\bar{x}_{t \mid t-1}=x_{t}$, it follows also that the optimal control cost is given by $J=\lim _{t \rightarrow \infty} \mathbb{E}\left[\left(\tilde{x}_{t}\right)^{2}\right]=$ $p+\sigma_{v}^{2}$.

Next, we can derive the modified algebraic Riccati equation (MARE) satisfied by $p$, given as follows:

$$
\begin{aligned}
p & =\left(1-\epsilon_{\gamma}\right) a^{2}(1-k)^{2} p+\epsilon_{\gamma} a^{2} p+\sigma_{w}^{2} \\
& +\left(1-\epsilon_{\gamma}\right) k^{2}\left(p_{\infty}^{t x}+\sigma_{n}^{2}\right) a^{2} \\
& +2\left(1-\epsilon_{\gamma}\right) k(1-k) a^{2} p_{\infty}^{t x}
\end{aligned}
$$

where we have used the fact that $\mathbb{E}\left[\tilde{x}_{t \mid t}^{t x} \tilde{x}_{t}\right]=p_{\infty}^{t x}$. By equating the corresponding matrix elements of the left hand side with the right hand side of (12), we obtain (after some algebraic manipulations)

$$
p=\frac{\sigma_{w}^{2}+a^{2} k\left(1-\epsilon_{\gamma}\right) p_{\infty}^{t x}\left(2-k\left(1+\frac{1}{\Lambda}\right)\right)}{\left[1-\epsilon_{\gamma} a^{2}-a^{2}\left(1-\epsilon_{\gamma}\right)\left((1-k)^{2}+\frac{k^{2}}{\Lambda}\right)\right]}
$$

We can now compute the optimal cost for the full channel feedback case.

Theorem 2: With perfect causal knowledge of packet loss sequence $\Gamma_{t}$ at the transmitter, the optimal controller gain and the optimal filter gain at the receiver are given by $l^{\text {opt }}=-a$ (dead-beat control) and $k^{o p t}=\frac{1}{1+1 / \Lambda}$ The corresponding steady state control cost $\lim _{t \rightarrow \infty} \mathbb{E}\left[y_{t}^{2}\right]$ is given by $J_{i f}^{o p t}=$ $p^{o p t}+\sigma_{v}^{2}$, where

$$
p^{o p t}=\frac{a^{2}\left(1-\epsilon_{\gamma}\right) \frac{p_{\infty}^{t x}}{1+1 / \Lambda}+\sigma_{w}^{2}}{\left(1-\epsilon_{\gamma} a^{2}\right)-\frac{a^{2}\left(1-\epsilon_{\gamma}\right)}{\Lambda+1}}
$$

which is finite under the assumption that SQNR $\Lambda$ is larger than a critical threshold $\Lambda^{o p t}=\frac{a^{2}-1}{1-\epsilon_{\gamma} a^{2}}$ with $\epsilon_{\gamma}<\frac{1}{a^{2}}$.

It is instructive to compare this result against the corresponding analysis for measurement forwarding performed in [25], where the sensor simply forwards each measurement $y_{t}$ as a packet, and hence does not need to know packet acknowledgement process. In this case, it was shown in [25] that the optimal controller gain is the sane dead-beat control $l^{o p t}=-a$, and the critical SQNR threshold for stabilization is also $\Lambda^{\text {opt }}=\frac{a^{2}-1}{1-\epsilon_{\gamma} a^{2}}$ with $\epsilon_{\gamma}<\frac{1}{a^{2}}$., but the optimal control cost is given by $J_{m f}^{\text {opt }}=\bar{p}+\sigma_{v}^{2}$ where $\bar{p}$ satisfies the following quadratic equation

$$
\bar{p}=a^{2} \bar{p}+\sigma_{w}^{2}-\frac{\left(1-\epsilon_{\gamma}\right)}{1+1 / \Lambda} \frac{\bar{p}}{\bar{p}+\sigma_{v}^{2}}
$$

In this case, the optimal constant gain for the receiver filter is given by $\bar{k}=\frac{1}{1+1 / \Lambda} \frac{\bar{p}}{\bar{p}+\sigma_{v}^{2}}$. Further details can be found in [25]. One can therefore draw the conclusion that with innovation forwarding, one cannot gain in terms of the critical SQNR threshold, in that it does not enlarge the stability margin. However, the optimal constant gain for the receiver filter is given by a simple expression, which is also the same for innovation forwarding with full channel feedback for remote estimation as studied in [19]. This is expected because the optimal control design with innovation forwarding also employs the same constant gain receiver filter as remote estimation with innovation forwarding, as explained in Section III, where we established the optimality of innovation forwarding at the transmitter when restricted to linear encoding.

The optimality of innovation forwarding is reflected not in the enlargement of stability margin, but in terms of performance, that is, with a reduced control cost $J_{i f}^{\text {opt }}$ which is smaller than $J_{m f}^{o p t}$, as guaranteed by Theorem 1 .

In the remaining part of the Section we provide an analysis of the difference $J_{m f}^{o p t}-J_{i f}^{o p t}$ which also reveals that it is and increasing function of the packet loss probability $\epsilon_{\gamma}$.

Let us first introduce a few notations. Define $b_{p}=\sigma_{v}^{2}\left(a^{2}-\right.$ 1) $+\sigma_{w}^{2}, c_{p}=\sigma_{w}^{2} \sigma_{v}^{2}$, and $\delta_{p}=\frac{a^{2}\left(1-\epsilon_{\gamma}\right)}{1+\frac{1}{\Lambda}}-\left(a^{2}-1\right)$. Note that when $\Lambda>\Lambda^{o p t}=\frac{a^{2}-1}{1-\epsilon_{\gamma} a^{2}}$ with $\epsilon_{\gamma}<\frac{1}{a^{2}}$., $\delta_{p}>0$. Based on the above notation, it is easy to show that $\bar{p}$, as a solution of (15) satisfies $\bar{p}=\frac{1}{2 \delta_{p}}\left(b_{p}+\sqrt{b_{p}^{2}+4 c_{p} \delta_{p}}\right)$, whereas $p^{o p t}$ from (14) satisfies

$$
p^{o p t}=\frac{1}{\delta_{p}}\left(\left(\delta_{p}+a^{2}-1\right) \frac{\sigma_{v}^{2} \bar{p}_{\infty}^{t x}}{\sigma_{v}^{2}+\bar{p}_{\infty}^{t x}}+\sigma_{w}^{2}\right)
$$

where recall that $\bar{p}_{\infty}^{t x}$ is the steady-state transmitter prediction error variance, given by $\left.\bar{p}_{\infty}^{t x}=\frac{1}{2}\left(b_{p}+\sqrt{(} b_{p}^{2}+4 c_{p}\right)\right)$. We also have the difference between the optimal control cost with measurement forwarding and innovation forwarding as $J_{m f}^{o p t}-J_{i f}^{o p t}$, given by $\bar{p}-p^{o p t}=\tilde{p}_{d i f f}$. Using the expressions for $\bar{p}$ and $p^{o p t}$ above, one can show the following:

$$
\begin{aligned}
\frac{d \tilde{p}_{d i f f}}{d \delta_{p}} & =\frac{1}{2 \delta_{p}^{2}}\left[-b_{p}+2\left(\sigma_{w}^{2}+\left(a^{2}-1\right) p_{\infty}^{t x}\right.\right. \\
& \left.-\frac{b_{p}^{2}+2 c_{p} \delta_{p}}{\sqrt{b_{p}^{2}+4 c_{p} \delta_{p}}}\right] \\
& <\frac{1}{2 \delta_{p}^{2}}\left[-b_{p}+2\left(\sigma_{w}^{2}+\left(a^{2}-1\right) \sigma_{v}^{2}\right.\right. \\
& \left.-\frac{b_{p}^{2}+2 c_{p} \delta_{p}}{\sqrt{b_{p}^{2}+4 c_{p} \delta_{p}}}\right] \\
& =\frac{1}{2 \delta_{p}^{2}}\left[b_{p}-\frac{b_{p}^{2}+2 c_{p} \delta_{p}}{\sqrt{b_{p}^{2}+4 c_{p} \delta_{p}}}\right]<0
\end{aligned}
$$

where the first inequality follows from the fact that $p_{\infty}^{t x}=$ $\frac{\sigma_{v}^{2} \bar{p}_{\infty}^{t x}}{\sigma_{v}^{2}+\bar{p}_{\infty}^{t x}}<\sigma_{v}^{2}$, and the second inequality follows from simple algebra, and we make use of the definition of $b_{p}$. Recall that $\delta_{p}>0$ when $\Lambda>\Lambda^{\text {opt }}=\frac{a^{2}-1}{1-\epsilon_{\gamma} a^{2}}$ with $\epsilon_{\gamma}<\frac{1}{a^{2}}$. It is also obvious that $\delta_{p}$ is maximum when $\epsilon_{\gamma}=0$ (no 
packet loss) and $\Lambda=\infty$ (no quantization noise). Therefore the maximum value of $\delta_{p}=1$. It follows from (17) that $\tilde{p}_{\text {diff }}$ is a decreasing function of $\delta_{p}$, and attains its minimum value when $\delta_{p}=1$. This minimum value is given by (from simple substitution in the expressions of $\bar{p}$ and $p^{\text {opt }}$ above) $\bar{p}_{\infty}^{t x}-\left(a^{2} p_{\infty}^{t x}+\sigma_{w}^{2}\right)=0$, since $\bar{p}_{\infty}^{t x}, p_{\infty}^{t x}$ are the transmitter steady-state prediction error and filtering error variances, respectively. Thus we have the following result.

Theorem 3: The difference between the optimal control cost with measurement forwarding and innovation forwarding with perfect acknowledgements, $J_{m f}^{o p t}-J_{i f}^{o p t}$, is an increasing function of $\delta_{p}=\frac{a^{2}\left(1-\epsilon_{\gamma}\right)}{1+\frac{1}{\Lambda}}-\left(a^{2}-1\right)$ as long as $\Lambda>\frac{a^{2}-1}{1-\epsilon_{\gamma} a^{2}}$ with $\epsilon_{\gamma}<\frac{1}{a^{2}}$.. If the SQNR $\Lambda>\frac{a^{2}-1}{1-\epsilon_{\gamma} a^{2}}$ is kept fixed, then $J_{m f}^{o p t}-J_{i f}^{o p t}$ is an increasing function of the packet loss probability $\epsilon_{\gamma}$. In addition, $J_{m f}^{o p t}=J_{i f}^{o p t}$ precisely when $\delta_{p}=1$, or when there is no packet loss and the SQNR is infinity so that there is no quantization noise.

The above theorem clearly quantifies the difference in the control performance between the measurement forwarding and the innovation forwarding strategies.

\section{ENCODER-Decoder Design With IMPERfECT Channel FeEdBack}

In this section, we discuss a few suboptimal strategies for encoder-decoder design when the packet acknowledgements are erased randomly according to a Bernoulli process $\nu_{t} \in$ $\{0,1\}$ where $P\left(\nu_{t}=0\right)=\epsilon_{\nu}$. Hence, the transmitter receives $\hat{\gamma}_{t}=\gamma_{t}$ when $\nu_{t}=1$, but does not know $\gamma_{t}$ when $\nu_{t}=0$. We focus on the innovation forwarding scenario since this is the optimal strategy with perfect channel feedback. However, since the transmitter does not know $\gamma_{t}$ whenever $\nu_{t}=0$, it also does not know the control input $u_{t}$ generated by the controller at the receiver end. Therefore, we focus on three different strategies for estimating $\gamma_{t}$ at the transmitter whenever $\nu_{t}=0$, see Figure 2. Below, we describe these three strategies without providing a detailed theoretical analysis, which is the focus of ongoing work. The first two strategies do not use the output $y_{t}$ to estimate $\gamma_{t}$, while the third one explicitly use it, resulting in a nonlinear scheme. In all of these strategies, we only design the encoder. The decoder and the controller are left as the constant gain filter with $k^{o p t}$ and $l^{o p t}$, respectively. In what follows, we assume $b=c=1$ for simplicity, as before.

\section{Randomized policy}

In this case, the transmitter replaces $\gamma_{t}$ by $\hat{\gamma}_{t}$ whenever $\nu_{t}=0$ by the following rule: $P\left(\hat{\gamma}_{t}=1 \mid \nu_{t}=0\right)=\beta$ and $P\left(\hat{\gamma}_{t}=0 \mid \nu_{t}=0\right)=1-\beta$, where $0 \leq \beta \leq 1$. The value of $\beta$ is optimized by exhaustive search such that $J=\lim _{t \rightarrow \infty} \mathbb{E}\left(y_{t}^{2}\right)$ is minimized. Of course, $\hat{\gamma}_{t}=\gamma_{t}$ when $\nu_{t}=1$.

\section{Soft estimation}

In this case, whenever $\nu_{t}=0, \gamma_{t}$ is replaced by $\mathbb{E}\left[\gamma_{t}\right]=$ $\epsilon_{\gamma}$ as suggested in [15], and when $\nu_{t}=1, \hat{\gamma}_{t}=\gamma_{t}$, as before. Note that for both the Randomized policy and Soft

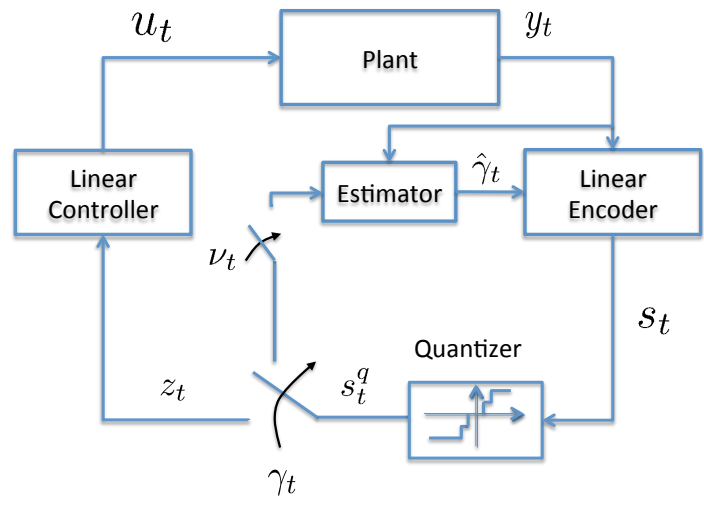

Fig. 2. Control scheme with imperfect feedback: when $\nu_{t}=0$ an estimate of the loss sequence is produced by the "Estimator' block. Three strategies are considered: (1) Randomized policy $P\left[\hat{\gamma}_{t}=1 \mid \nu_{t}=0\right]=\beta$, (2) Soft estimation: $\hat{\gamma}_{t}=\mathbb{E}\left[\gamma_{t}\right]=\epsilon_{\gamma}$, (3) Non-linear estimator: $\hat{\gamma}_{t}=$ $\arg \min _{\gamma \in\{0,1\}}\left(y_{t+1}-\hat{y}_{t+1}(\gamma)\right)^{2}$.

estimation policy, the control cost can be obtained via an average covariance analysis of the matrix $\bar{P}=\mathbb{E}\left[\xi_{t} \xi_{t}^{\prime}\right]$, where $\xi_{t}=\left[x_{t} \hat{x}_{t \mid t-1}^{t x}\right]^{\prime}$, and the control cost is given by $J=\mathbb{E}\left[x_{t}^{2}\right]+$ $\sigma_{v}^{2}=\bar{p}_{11}+\sigma_{v}^{2}$, with $\bar{p}_{11}$ being the first diagonal element of $\bar{P}$. We leave this analysis and corresponding stability margin calculations for future work.

\section{Nonlinear encoder design}

In this case, we estimate $\gamma_{t}$ from the observed measurement $y_{t+1}$ at the transmitter by a simple distance-based rule, whenever $\nu_{t}=0$. Due to the dead beat controller, we have the control input $u_{t}=-a \bar{x}_{t \mid t}=-a k^{o p t} \gamma_{t}\left(\hat{x}_{t \mid t}^{t x}+n_{t}\right)$. Then one can predict a value of $y_{t+1}$ as a function of $\gamma_{t}$ as $y_{t+1}\left(\gamma_{t}\right)=a \hat{x}_{t \mid t}^{t x}-a k^{o p t} \gamma_{t}\left(\hat{x}_{t \mid t}^{t x}+n_{t}\right)$. We estimate $\gamma_{t}$ as $\hat{\gamma}_{t}=\arg \min _{\gamma \in\{0,1\}}\left(y_{t+1}-\hat{y}_{t+1}(\gamma)\right)^{2}$, similarly to [16]. Clearly, in this case, since $\hat{\gamma}_{t}$ is a nonlinear function of $y_{t+1}$, the encoder implements a nonlinear filter at the transmitter, implying that a steady state analysis of the feedback control performance will be difficult and indeed we leave it for ongoing work.

\section{NUMERICAL RESULTS}

In this section we present some numerical results on a comparative study of the various encoder-decoder design strategies discussed in the earlier sections. In particular, we consider a linear dynamical system with $a=1.25, b=c=1$ and $\sigma_{w}^{2}=0.02, \sigma_{v}^{2}=0.2$ and feedback erasure probability $\epsilon_{\nu}=0.2$. We vary the forward channel (between sensor and decoder) packet loss probability between 0.1 and 0.4 , while the SQNR is chosen to be $\Lambda\left(\epsilon_{\gamma}\right)=1.2 \Lambda^{\text {opt }}$ where $\Lambda^{\text {opt }}=$ $\frac{a^{2}-1}{1-\epsilon_{\gamma} a^{2}}$. Figure 3 illustrates the control cost performance of the various encoding-decoding strategies discussed above for perfect and imperfect channel feedback with measurement and innovations forwarding. Note that for measurement forwarding at the transmitter, the channel feedback is irrelevant to the transmitter, whereas the strategy of innovation forwarding is optimal when there is perfect channel feedback. 


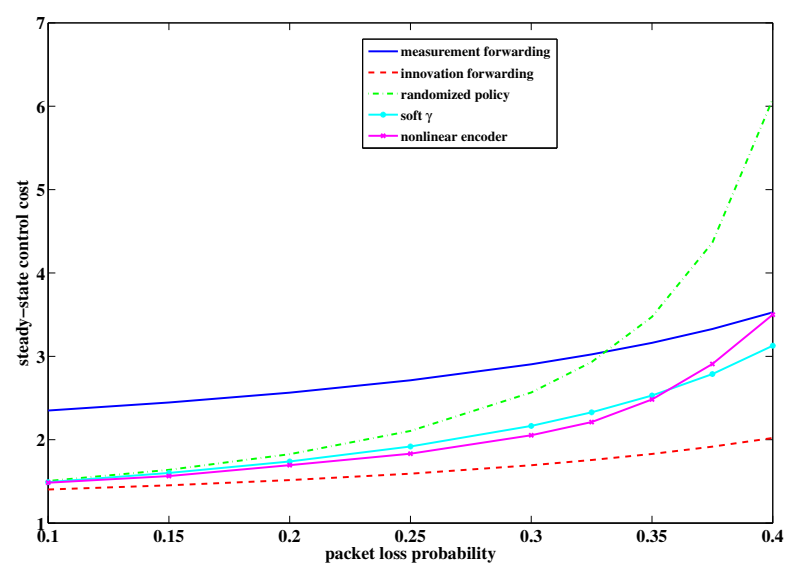

Fig. 3. Control cost comparison for various encoding-decoding strategies with measurement and innovation forwarding

It is clearly seen from the graph that innovation forwarding does substantially better than measurement forwarding for all packet loss probabilities. Amongst the suboptimal strategies for encoder design with imperfect channel feedback, it can be seen that the randomized policy performs the worst, whereas the soft $\gamma$ estimation and the nonlinear encoding strategies perform much better, with the nonlinear encoding strategy performing the best at lower packet loss probabilities. When the packet loss probability becomes reasonably high, the suboptimal schemes can perform poorly. It can also be seen that the randomized policy performs even poorer than measurement forwarding, implying that the randomized policy is not very useful when the packet loss probability becomes higher.

\section{CONCLUSIONS}

In this paper, we considered an unstable scalar linear dynamical system that operates over a channel that is prone to packet loss as well as quantization noise. We show that when there is perfect packet acknowledgement available at the transmitter, the optimal linear encoding strategy at the transmitter is to encode the innovations defined as the difference between the transmitter pre-processing Kalman filtered estimate and the receiver filter predicted estimate. For this strategy, we also find the optimal controller and the optimal constant gain receiver filter, which allows us to characterize the steady-state control cost. We also provide an analysis that quantitatively characterizes the difference between the control costs obtained via measurement forwarding at the transmitter and the optimal innovation forwarding strategy described above. For the case of imperfect channel feedback, we propose three suboptimal encoding strategies the performances of which are investigated via simulation studies. Future work will investigate the extension of these studies to the case of unstable vector systems and consider further theoretical performance analysis of the suboptimal strategies for the case of imperfect channel feedback.

\section{REFERENCES}

[1] J. H. Braslavsky, R. H. Middleton, and J. S. Freudenberg, "Feedback stabilization over signal-to-noise ratio constrained channels," in In Proc. of the 2004 Amer. Contr. Conf., June 2004, pp. 4903-4908.

[2] S. Tatikonda and S. Mitter, "Control under communication constraints," IEEE Transaction on Automatic Control, vol. 49, no. 7, pp. 1056-1068, July 2004.

[3] A. Sahai and S. Mitter, "The necessity and sufficiency of anytime capacity for control over a noisy communication link: Part I,' IEEE Transaction on Information Theory, 2006.

[4] N. Elia and J. Eisembeis, "Limitation of linear control over packet drop networks," in Proceedings of IEEE Conference on Decision and Control, vol. 5, Bahamas, December 2004, pp. 5152-5157.

[5] L. Schenato, B. Sinopoli, M. Franceschetti, K. Poolla, and S. Sastry, "Foundations of control and estimation over lossy networks," Proceedings of the IEEE, vol. 95, pp. 163-187, 2007.

[6] N. C. Martins and M. A. Dahleh, "Feedback control in the presence of noisy channels: Bode-like fundamental limitations of performance," IEEE Transactions on Automatic Control, vol. 52, p. 16041615, 2008.

[7] A. Matveev and A. Savkin, "The problem of LQG optimal control via a limited capacity communication channel," Systems and Control Letters, vol. 53, no. 1, pp. 51-64, 2004.

[8] K. Tsumura, H. Ishii, and H. Hoshina, "Tradeoffs between quantization and packet loss in networked control of linear systems," Automatica, vol. 45, no. 12, pp. 2963-2970, 2009.

[9] P. Minero, L. Coviello, and M. Franceschetti, "Stabilization over Markov feedback channels: The general case," Transactions on Automatic Control, vol. 58, no. 2, pp. 349-362, 2013.

[10] E. Silva and S. Pulgar, "Performance limitations for single-input LTI plants controlled over SNR constrained channels with feedback," Automatica, vol. 2, no. 49, pp. 540-547, 2013.

[11] V. Borkar and S. Mitter, Festschrift: Communications, Computation, Control and Signal Processing: A Tribute to Thomas Kailath. Kluwer/Springer-Verlag, 1997, ch. LQG Control with Communication Constraints.

[12] O. C. Imer, S. Yuksel, and T. Basar, "Optimal control of dynamical systems over unreliable communication links," Automatica, vol. 42, no. 9, pp. 1429-1440, September 2006.

[13] B. Sinopoli, L. Schenato, M. Franceschetti, K. Poolla, and S. Sastry, "Optimal linear lqg control over lossy networks without packet acknowledgment," Asian Journal of Control, vol. 10, pp. 3-13, 2008.

[14] M. Trivellato and N. Benvenuto, "State control in networked control systems under packet drops and limited transmission bandwidth," IEEE Transaction on Communications, vol. 58, no. 2, pp. 611-622, 2010.

[15] E. Garone, B. Sinopoli, and A. Casavola, "LQG control over lossy TCP-like networks with probabilistic packet acknowledgment," International Journal of Systems, Control and Communications, vol. 2, pp. 55-81, 2010.

[16] M. Epstein, L. Shi, and R. M. Murray, "Estimation schemes for networked control systems using udp-like communication," in Prooceedings of IEEE Conference on Decision and Control (CDC'07), Kos, Greece, 2007, pp. 3945 - 3951.

[17] V. Gupta, D. Spanos, B. Hassibi, and R. M. Murray, "Optimal LQG control across a packet-dropping link," Systems and Control Letters, vol. 56, no. 6, pp. 439-446, 2007.

[18] S. Chitode, Digital communications. Technical Publications, 2009.

[19] S. Dey, A. Chiuso, and L. Schenato, "Remote estimation with noisy measurements subject to packet loss and quantization noise," IEEE Trans. Control of Network Systems, vol. 1, pp. 204-217, 2013.

[20] A. Chiuso, N. Laurenti, L. Schenato, and A. Zanella, "LQG-like control of scalar systems over communication channels: The role of data losses, delays and SNR limitations," Automatica, 2014.

[21] D. Marco and D. Neuhoff, "The validity of the additive noise model for uniform scalar quantizers," IEEE Trans. Info. Theory, vol. 51, no. 5 , pp. 1739-1755, 2005.

[22] V. Goyal, "High-rate transform coding: how high is high, and does it matter?" in Proc. IEEE Intl. Symp. Info. Theory (ISIT), 2000.

[23] A. Leong, S. Dey, and G. Nair, "Quantized filtering schemes for multisensor linear state estimation: Stability and performance under high rate quantization," IEEE Trans. Sig. Proc., vol. 61, no. 15, pp. 3852$3865,2013$.

[24] S. Dey, A. Chiuso, and L. Schenato, "Linear encoder-decodercontroller design over channels with packet loss and quantization noise," University of Padova, Tech. Rep., 2015.

[25] F. Parise, L. D. Col, A. Chiuso, N. Laurenti, L. Schenato, and A. Zanella, "Impact of a realistic transmission channel on the performance of control systems," University of Padova, Tech. Rep., 2013. 